\title{
Low flow velocity in the middle cerebral artery predicting infarction after bypass surgery in adult moyamoya disease
}

\author{
*Hoyeon Cho, MD,, Kyung II Jo, MD, ${ }^{2}$ Jua Yu, RN, ${ }^{1}$ Je Young Yeon, MD, MSc, ${ }^{1}$ \\ Seung-Chyul Hong, MD, PhD, ${ }^{1}$ and Jong Soo Kim, MD, PhD1
}

1Department of Neurosurgery, Samsung Medical Center, Sungkyunkwan University School of Medicine, Seoul; and 'Department of Neurosurgery, Hana General Hospital, Cheongju, Korea

\begin{abstract}
OBJECTIVE Direct and indirect bypass surgeries are recognized as the most effective treatments for preventing further stroke in adults with moyamoya disease (MMD). However, the risk factors for postoperative infarction after bypass surgery for MMD are not well established. Therefore, the objective of this study was to investigate the risk factors for postoperative infarction. In particular, the authors sought to determine whether transcranial Doppler (TCD) ultrasonography measurements of mean flow velocity (MFV) in the middle cerebral artery (MCA) could predict postrevascularization infarction.
\end{abstract}

\begin{abstract}
METHODS The medical records of patients with MMD who underwent direct bypass surgery at the authors' institution between July 2012 and April 2015 were reviewed. The MFV in the MCA was measured with TCD ultrasonography and categorized as high (> $80 \mathrm{~cm} / \mathrm{sec}$ ), medium (40-80 cm/sec), and low $(<40 \mathrm{~cm} / \mathrm{sec})$. Postoperative MRI, including diffusion-weighted imaging, was performed for all patients within a week of their surgery. Angiographic findings were classified according to the Suzuki scale. Postrevascularization infarction was defined as any diffusion restriction on postoperative MRI scans. Postoperative neurological status was assessed through a clinical chart review, and the modified Rankin Scale was used to evaluate clinical outcomes.
\end{abstract}

RESULTS Of 43 hemispheres in which bypass surgery for MMD was performed, 11 showed postrevascularization infarction. Ten of these hemispheres had low MFV and 1 had medium MFV in the ipsilateral MCA. In both univariate and multivariate analyses, a low MFV was associated with postrevascularization infarction (adjusted OR 109.2, 95\% Cl 1.9-6245.3). A low MFV was also statistically significantly associated with more advanced MMD stage $(p=0.02)$.

CONCLUSIONS A low MFV in the ipsilateral MCA may predict postrevascularization infarction. Bypass surgery for MMD appears to be safe in early-stage MMD. Results of TCD ultrasonography provide clinical data on the hemodynamics in MMD patients before and after revascularization.

https://thejns.org/doi/abs/10.3171/2016.3.JNS152256

KEY WORDS transcranial Doppler; moyamoya disease; cerebral infarction; risk factors; stroke; hemodynamics; vascular disorders

$\mathrm{M}$ OYAMOYA disease (MMD) is a rare steno-occlusive cerebrovascular disease with a relatively high incidence in East Asia. ${ }^{1}$ Direct and indirect revascularizations are recognized as the only effective treatments to prevent further stroke in MMD..$^{1,9,10}$ However, several complications, such as intracranial hemorrhage, acute cerebral infarction, hyperperfusion syndrome, recurrent transient ischemic attack, and other surgical adverse events can occur after revascularization. ${ }^{5,6,20}$ Among these complications, acute cerebral infarction is both common and the cause of irreversible neurological deficits and even death. However, the risk factors and mechanism of postrevascularization infarction have not been clearly identified. ${ }^{3,5}$ In this study, baseline characteristics in patients with MMD, including results of transcranial Doppler (TCD) ultrasonography, were analyzed to identify risk factors for postrevascularization infarction in MMD patients who underwent direct revascularization surgery.

ABBREVIATIONS DWI = diffusion-weighted imaging; MCA = middle cerebral artery; MFV = mean flow velocity; MMD = moyamoya disease; mRS = modified Rankin Scale; STA = superficial temporal artery; $T C D=$ transcranial Doppler.

SUBMITTED September 26, 2015. ACCEPTED March 8, 2016.

INCLUDE WHEN CITING Published online June 3, 2016; DOI: 10.3171/2016.3.JNS152256.

* Drs. Cho and Jo contributed equally to this work. 


\section{Methods}

\section{Patient Selection and Characteristics}

We analyzed prospectively collected data in a database of patients with MMD who were 20 years or older and seen at our institution between July 2012 and April 2015. The inclusion criteria for this study were angiographically confirmed MMD in patients who had undergone direct or combined (i.e., direct plus indirect) bypass surgery. Patients treated solely with an indirect bypass surgery were excluded as were patients without available preoperative TCD data $(n=13)$. Thirty-six patients (representing 43 treated hemispheres) met the inclusion criteria and were included in this study.

The patients' characteristics, including sex, age, and medical history, which included hypertension, family history of MMD, current smoking, and preoperative status, were retrospectively reviewed. The data were correlated with preoperative radiological images and the presence of recent ( $<3$ months) ischemic symptoms.

\section{Data Collection and Analysis}

Angiographic findings in the patients were classified according to the Suzuki and Takaku angiographic staging method involving digital subtracted angiography. ${ }^{18}$ Perfusion status was evaluated with basal and acetazolamide brain SPECT. Basal and acetazolamide brain SPECT scanning procedures with ${ }^{99 \mathrm{~m}} \mathrm{Tc}-$ hexamethylpropyleneamine oxime were used to evaluate basal perfusion and vascular reserve. Two experienced nuclear medicine physicians qualitatively interpreted the SPECT images. The degree of perfusion abnormality was graded as mild, moderate, or severe.

The TCD studies were performed with a standard 2-MHz, pulsed-wave ultrasound system (Pioneer TC 8080; Nicolet Vascular) with a handheld transducer in a range-gated, pulsed-wave mode in a single laboratory. All examiners followed the same protocol. The mean flow velocity (MFV) in the middle cerebral artery (MCA) was categorized according to the median value of MFV as follows: low MFV $<40 \mathrm{~cm} / \mathrm{sec}$, medium MFV $40-80 \mathrm{~cm} /$ sec, and high MFV $>80 \mathrm{~cm} / \mathrm{sec}$. The TCD studies were conducted by 3 examiners and were interpreted by 2 experienced neurologists.

All patients underwent direct bypass surgery involving the superficial temporal artery (STA) to the MCA or combined surgery comprising direct bypass (STA-MCA) and encephaloduroarteriosynangiosis involving both the frontal and parietal branches of the STA if applicable. In 40 surgeries (93.0\%), the bypass point was the $\mathrm{M}_{4}$ segment of the MCA in the ipsilateral frontal cortex, and in $3(7.0 \%)$, the bypass point was the $\mathrm{M}_{4}$ segment of the MCA in the ipsilateral temporal cortex. All surgical procedures were performed under general anesthesia and with neurophysiological monitoring, including motor evoked and somatosensory evoked potential monitoring.

Postoperative MRI, including diffusion-weighted imaging (DWI), was performed and images reviewed for all patients within a week after surgery. MRI was performed at $3 \mathrm{~T}$ (Achieva; Philips Healthcare) with an 8-channel sensitivity-encoding head coil. Postrevascularization in- farction was defined as any diffusion restriction on postoperative MRI scans. These were divided into 3 groups according to the size, number, and location of lesions on the DWI scan. The diameters of the diffusion-restricting lesions were categorized as small $(<15 \mathrm{~mm})$, large $(\geq 15$ $\mathrm{mm}$ ), and territorial. ${ }^{2}$ Locations of infarctions were categorized as being in a concordant area or not, depending on whether the lesions identified on DWI scans were located in an area corresponding to the recipient vessel. All postoperative imaging results were reviewed by 2 independent examiners (1 radiologist and 1 neurosurgeon) who were blinded to the preoperative evaluation.

Postoperative neurological status was assessed through a clinical chart review. Transient neurological deficits were defined as neurological deficits that resolved within 72 hours. The modified Rankin Scale (mRS) was used to evaluate clinical outcomes; a poor outcome was defined as an $\mathrm{mRS}$ score $>2$.

\section{Statistical Analysis}

Statistical analyses were performed with SPSS version 22.0 (IBM Corp.). Univariate statistical analyses with a chi-square test or Fisher exact test were performed to assess the association of clinical, radiological, and TCD ultrasonography results with postoperative infarction. A trend analysis was performed with a linear-by-linear association model in a 2-sided chi-square test to assess whether increased cerebral angiographic staging values were statistically significantly associated with MFV measured with TCD ultrasonography. A multivariate analysis was performed with a multiple logistic regression analysis.

\section{Results}

The baseline characteristics of the patients in the present study are given in Table 1. These characteristics were similar among patients grouped according to MFV in the MCA, except for Suzuki stage. The median age of the patients included in this analysis was 35 years (range 20-64 years), and $16(37.2 \%)$ of the patients were men. Thirty-six patients underwent an indirect bypass combined with direct bypass. A low MFV was more prevalent in advancedstage MMD than in the other Suzuki stages $(p=0.002)$ (Fig. 1).

Hemodynamic status did not correlate with MFV in the MCA in this patient cohort. Seven patients had symptomatic hyperperfusion after revascularization. Of these patients, 6 had low MFV, and 1 had medium MFV. There were no symptomatic hemorrhages, and none of the hyperperfusions resulted in permanent neurological deficits.

In $11(25.6 \%)$ of 43 hemispheres, postrevascularization infarctions occurred (Table 2). Seven of these lesions were small, 4 were large, and no territorial infarctions were observed. We noted 8 transient neurological deficits, 2 asymptomatic deficits, and 1 case of prolonged weakness resulting from these lesions. Transient neurological deficits comprised 5 cases of dysarthria, 2 of weakness, 1 of both dysarthria and weakness, and 1 of lethargy. All transient neurological symptoms improved within a few hours after infarction and returned to normal within 48 hours. One prolonged neurological deficit resulted in an 
TABLE 1. Baseline characteristics grouped according to MFV in the ipsilateral $M A^{*}$

\begin{tabular}{|c|c|c|c|c|}
\hline Characteristic & $\begin{array}{l}\text { High MFV } \\
(n=7)\end{array}$ & $\begin{array}{c}\text { Medium } \\
\text { MFV }(n=12)\end{array}$ & $\begin{array}{c}\text { Low MFV } \\
(n=24)\end{array}$ & $p$ Value \\
\hline $\mathrm{M} / \mathrm{F}$ ratio & $2: 5$ & $3: 9$ & $11: 13$ & 1.000 \\
\hline $\begin{array}{l}\text { Median age in yrs } \\
\text { (range) }\end{array}$ & $35(27-64)$ & $35(26-54)$ & $36(20-58)$ & 0.914 \\
\hline Hypertension & 5 & 3 & 13 & 1.000 \\
\hline Current smoking & 3 & 2 & 6 & 0.648 \\
\hline Family $\mathrm{Hx}$ of MMD & 1 & 1 & 1 & 0.447 \\
\hline Initial presentation & & & & 0.915 \\
\hline Hemorrhage & 2 & 2 & 4 & \\
\hline Infarction & 2 & 3 & 7 & \\
\hline TIA & 2 & 6 & 12 & \\
\hline Atypical & 0 & 1 & 1 & \\
\hline Incidental & 1 & 0 & 0 & \\
\hline $\begin{array}{l}\text { Recent ischemic } \\
\text { event }\end{array}$ & 5 & 8 & 15 & 0.683 \\
\hline Suzuki stage & & & & 0.002 \\
\hline I & 2 & 0 & 0 & \\
\hline II & 0 & 1 & 1 & \\
\hline III & 4 & 10 & 11 & \\
\hline IV & 1 & 1 & 11 & \\
\hline V & 0 & 0 & 1 & \\
\hline PCA involvement & 4 & 1 & 10 & 0.473 \\
\hline
\end{tabular}

$\mathrm{Hx}=$ history; $\mathrm{PCA}=$ posterior cerebral artery; $\mathrm{TIA}=$ transient ischemic attack.

* A total of 43 hemispheres were treated in 36 patients.

mRS score of 3 at the 3-month follow-up. Five (45.5\%) of the 11 infarctions were located in the concordant area, and all of these were small; the patients with these infarctions presented with symptoms of transient neurological deficits. The other 6 infarctions (54.5\%) occurred in a discordant area.

Of the hemispheres surgically treated in this study, 10 had low MFV in the MCA (i.e., an MFV of $<40 \mathrm{~cm} / \mathrm{sec}$ ), and 1 had medium MFV (i.e., an MFV of $40-80 \mathrm{~cm} / \mathrm{sec}$ ). According to the univariate analysis (Table 3), a low MFV was statistically significantly associated with postrevascularization infarction $(\mathrm{p}=0.01)$. Other patient variables, including age, hypertension, family history of MMD, current smoking, preoperative status, and recent ischemic events, were not associated with postrevascularization infarction $(p>0.05)$. The multivariate analysis indicated concordant results; in this analysis, a low MFV in the MCA was again statistically significantly associated with postoperative infarction (OR 109.2, 95\% CI 1.9-6245.3, $\mathrm{p}=0.02)$.

\section{Discussion}

Revascularization interventions are considered the only effective treatment of patients with MMD and can decrease the risk for further stroke by improving the hemodynamic status of the brain vasculature. ${ }^{4,8,20}$ Several previous studies have suggested that multiple ischemic episodes or the presence of preoperative infarction in MMD are correlated with perioperative ischemic com-

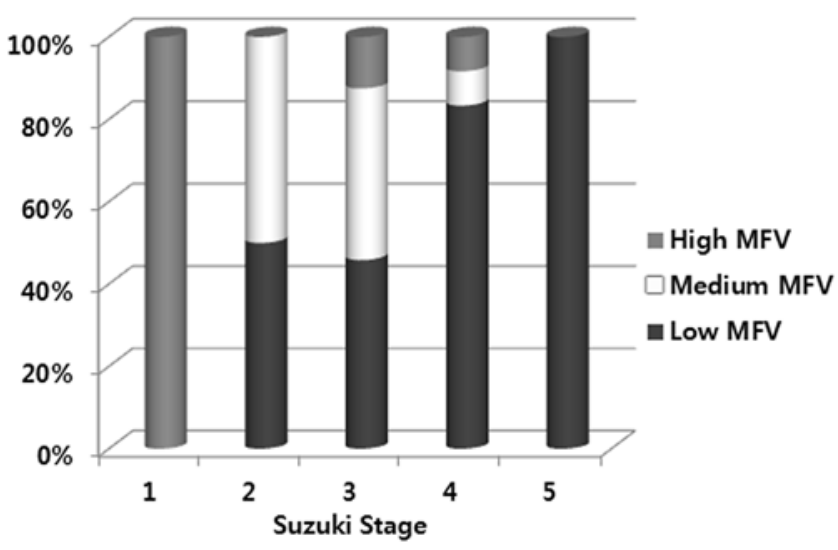

FIG. 1. Relationship of MFV in the MCA with Suzuki stage indicating an increase in the prevalence of low MFV in the MCA with more advancedstage MMD.

plications. ${ }^{8,12,17}$ In the present study, in $28(65.1 \%)$ of the hemispheres treated, recent ischemic events had occurred, and these events also showed a trend of being associated with perioperative ischemic complications; however, this trend did not reach statistical significance $(p=0.12)$.

In our series, the postrevascularization infarction rate was $25.6 \%$, considerably higher than infarction rates observed in previous reports. ${ }^{8,12,16,17}$ Nearly all of these infarctions resulted in only transient neurological deficits. Considering perioperative conditions under anesthesia for the patients with infarctions, 1 patient had a widened pulse pressure and another had a relatively low $\mathrm{CO}_{2}$ level (31 $\mathrm{mm} \mathrm{Hg}$ ) for 30 minutes during the operation, and no other findings such as significant hypotension were observed. None of the 2 patients had a postrevascularization infarction.

A previous study has reported that $22.6 \%$ of patients who underwent bypass surgery exhibited transient neurological deficits with local hypoperfusion..$^{15}$ We hypothesized that a high proportion of the transient ischemic attacks (approximately 75\%) may be due to diffusion-positive lesions. In the present study, immediate postprocedural MRI was performed in all cases, which may be an additional reason for the higher detection rate of postoperative infarctions. In addition, the relatively small number of patients may also be a reason for the observation of relatively higher rates postoperative ischemic changes in the present study. Therefore, we consider the incidence of postrevascularization infarction reported here acceptable.

Hayashi et al. have reported that watershed shift could be a reason for postoperative ischemia. ${ }^{7}$ Mukerji and colleagues showed that local hypoperfusion identified during perioperative periods might originate from competing blood flow. ${ }^{15}$ We hypothesized that preoperative hemodynamic status such as MFV in the MCA could predict a postoperative watershed shift and noted that most postrevascularization infarctions occurred in the low-MFV group. Moreover, more than half of the infarctions occurred at a site remote from the recipient bypass vessel. These findings suggest that postoperative watershed shifts could be predicted with preoperative TCD ultrasonography measurements of MFV in the MCA. Furthermore, our 


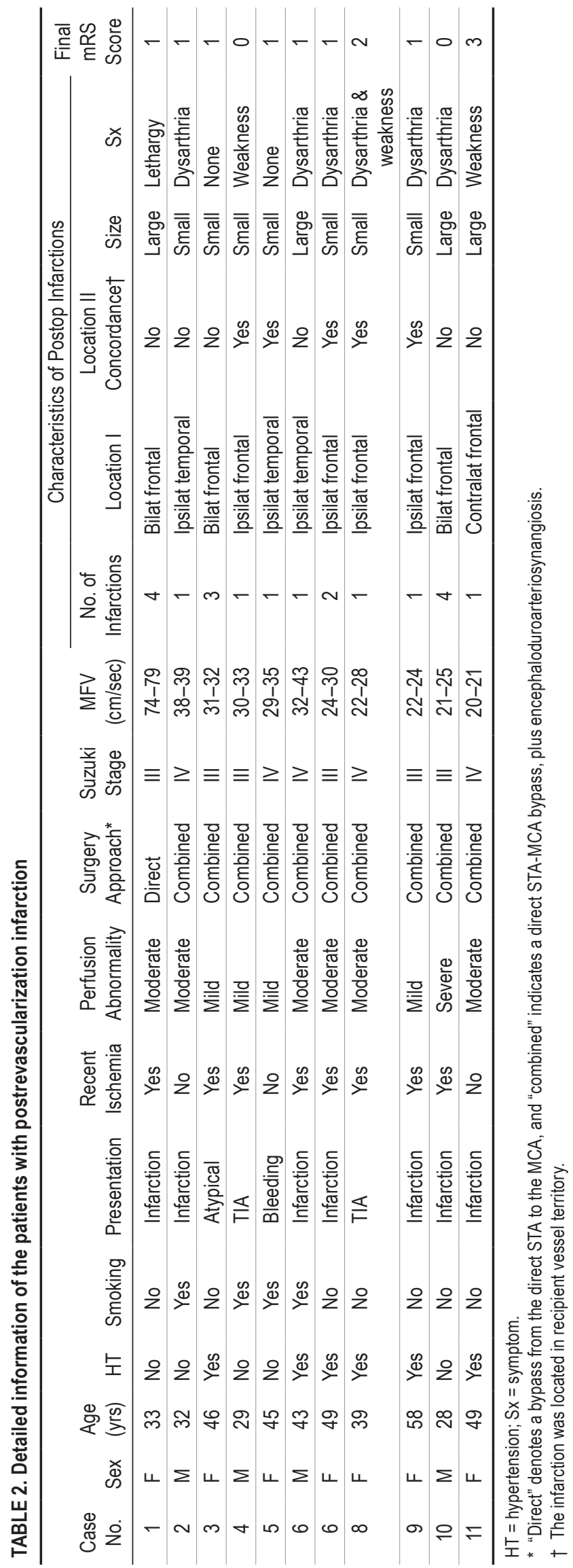

TABLE 3. Results of uni- and multivariate analyses of potential predictors of postrevascularization infarction

\begin{tabular}{lcccc}
\hline \multirow{2}{*}{ Factor } & $\begin{array}{c}\text { Univariate } \\
\text { Analysis }\end{array}$ & $\begin{array}{c}\text { Multivariate } \\
\text { Analysis }\end{array}$ & $\begin{array}{c}\text { Adjusted } \\
\text { OR }\end{array}$ & \multicolumn{1}{c}{$95 \% \mathrm{Cl}$} \\
\hline Age & 0.15 & 0.10 & 1.2 & $0.66-1.6$ \\
\hline Male & 1.00 & 0.61 & 0.48 & $0.03-8.0$ \\
\hline Hypertension & 0.74 & 0.10 & 0.04 & $0.52-1266.6$ \\
\hline Current smoking & 0.43 & 0.28 & 5.0 & $0.01-3.7$ \\
\hline Family Hx of MMD & 1.00 & 0.98 & 0.94 & $0.02-44.8$ \\
\hline Initial presentation & 0.74 & 0.88 & NA & NA \\
\hline Recent ischemic & 0.72 & 0.12 & 14.3 & $0.52-333.3$ \\
$\quad$ event & & & & \\
\hline Low MFV & 0.01 & $0.02 *$ & 109.2 & $1.9-6245.3$ \\
\hline Suzuki stage & 0.70 & 0.95 & 1.1 & $0.06-21.6$ \\
\hline PCA involvement & 0.47 & 0.57 & 2.6 & $0.02-9.7$ \\
\hline NA = not available. & & & & \\
* Statistically significant $p$ value. & & & \\
& & & & \\
\hline
\end{tabular}

observations indicated that infarctions in remote sites were larger and more clinically significant than in nonremote sites. In addition, 6 of 7 cases of hyperperfusion occurred in the low-MFV group. The findings of the present study further suggest that bypass surgery for MMD may have a higher risk for hemodynamic complications in patients with advanced MMD and low MFV in the MCA.

To the best of our knowledge, only a few studies have investigated MCA flow velocity in patients with MMD. ${ }^{13,14,19}$ These studies have shown that the MFV in the MCA peaks at Suzuki Stage II or III and decreases with more advanced stages. Our data showed similar findings for advanced-stage (Suzuki Stage IV or V) MMDs, but high MFV peaked at an earlier MMD stage (i.e., Suzuki Stage I). The results of our studies and those of previous ones therefore suggest that TCD ultrasonography measurements of MFV in the MCA may be an adjunctive tool for determining the Suzuki stage. However, because of small cohort sizes, none of the studies provided normalized MFV data in the MMD patients. To set optimal guidelines for clinical use of data on MFV in the MCA in patients with MMD, such as for predicting the risk for postrevascularization infarction, additional studies are required to determine the MFV threshold and identify other relevant criteria. Our data showed that MFV in the MCA was not associated with SPECT perfusion status, and this lack of an association may be due to profuse collateral vessels in patients with MMD.

This study had some limitations, including a relatively small patient cohort and a retrospective study design. The relatively high proportion of postoperative complications represents additional limitations of this study. Future prospective studies with larger cohorts may provide more conclusive evidence that MFV in the MCA is a reliable marker for predicting postoperative complications after bypass surgery for MMD. Nonetheless, we note that we observed a significantly increased association of postrevascularization infarction with low MFV. The findings 
of this study therefore provide useful information that requires further clinical validation. Examinations with TCD ultrasonography may yield reliable MFV data, although its accuracy may be affected by the angle between the probe and blood vessel..$^{11}$ Therefore, the tortuosity and direction of the MCA may have influenced the results of this study. We note the involvement of multiple examiners as another limitation of the present study, but we also stress that all TCD ultrasonography examinations were performed according to a standard protocol and were interpreted by 2 experienced neurologists. Therefore, we consider the effect of this limitation minor in this study.

\section{Conclusions}

A low MFV in the MCA may predict postrevascularization infarction after bypass surgery for MMD. A direct bypass can be performed more safely in early-stage MMD with high MFV in the MCA. Results of TCD ultrasonography provide useful clinical data on the hemodynamics in MMD patients in the pre- and postrevascularization periods.

\section{References}

1. Abla AA, Gandhoke G, Clark JC, Oppenlander ME, Velat GJ, Zabramski JM, et al: Surgical outcomes for moyamoya angiopathy at Barrow Neurological Institute with comparison of adult indirect encephaloduroarteriosynangiosis bypass, adult direct superficial temporal artery-to-middle cerebral artery bypass, and pediatric bypass: 154 revascularization surgeries in 140 affected hemispheres. Neurosurgery 73:430-439, 2013

2. Adams HP Jr, Bendixen BH, Kappelle LJ, Biller J, Love $\mathrm{BB}$, Gordon DL, et al: Classification of subtype of acute ischemic stroke. Definitions for use in a multicenter clinical trial. TOAST. Trial of Org 10172 in Acute Stroke Treatment. Stroke 24:35-41, 1993

3. Bai J, Zhao YL, Wang R, Zhang D, Wang S, Zhao JZ, et al: Regional cerebral perfusion and ischemic status after standard superficial temporal artery-middle cerebral artery (STA-MCA) bypass surgery in ischemic cerebrovascular disease. Childs Nerv Syst 28:579-586, 2012

4. Cho WS, Kim JE, Kim CH, Ban SP, Kang HS, Son YJ, et al: Long-term outcomes after combined revascularization surgery in adult moyamoya disease. Stroke 45:3025-3031, 2014

5. Funaki T, Takahashi JC, Takagi Y, Kikuchi T, Yoshida K, Mitsuhara T, et al: Unstable moyamoya disease: clinical features and impact on perioperative ischemic complications. $\mathbf{J}$ Neurosurg 122:400-407, 2015

6. Fung LW, Thompson D, Ganesan V: Revascularisation surgery for paediatric moyamoya: a review of the literature. Childs Nerv Syst 21:358-364, 2005

7. Hayashi T, Shirane R, Fujimura M, Tominaga T: Postoperative neurological deterioration in pediatric moyamoya disease: watershed shift and hyperperfusion. J Neurosurg Pediatr 6:73-81, 2010

8. Hyun SJ, Kim JS, Hong SC: Prognostic factors associated with perioperative ischemic complications in adult-onset moyamoya disease. Acta Neurochir (Wien) 152:1181-1188, 2010

9. Kazumata K, Ito M, Tokairin K, Ito Y, Houkin K, Nakayama $\mathrm{N}$, et al: The frequency of postoperative stroke in moyamoya disease following combined revascularization: a single-university series and systematic review. J Neurosurg 121:432-440, 2014

10. Kohno K, Oka Y, Kohno S, Ohta S, Kumon Y, Sakaki S: Cerebral blood flow measurement as an indicator for an indirect revascularization procedure for adult patients with moyamoya disease. Neurosurgery 42:752-758, 1998

11. Krejza J, Mariak Z, Babikian VL: Importance of angle correction in the measurement of blood flow velocity with transcranial Doppler sonography. AJNR Am J Neuroradiol 22:1743-1747, 2001

12. Kuroda S, Kamiyama H, Abe H, Asaoka K, Mitsumori K: Temporary neurological deterioration caused by hyperperfusion after extracranial-intracranial bypass-case report and study of cerebral hemodynamics. Neurol Med Chir (Tokyo) 34:15-19, 1994

13. Kwag HJ, Jeong DW, Lee SH, Kim DH, Kim J: Intracranial hemodynamic changes during adult moyamoya disease progression. J Clin Neurol 4:67-74, 2008

14. Lee YS, Jung KH, Roh JK: Diagnosis of moyamoya disease with transcranial Doppler sonography: correlation study with magnetic resonance angiography. J Neuroimaging 14:319323,2004

15. Mukerji N, Cook DJ, Steinberg GK: Is local hypoperfusion the reason for transient neurological deficits after STA-MCA bypass for moyamoya disease? J Neurosurg 122:90-94, 2015

16. Ohue S, Kumon Y, Kohno K, Watanabe H, Iwata S, Ohnishi T: Postoperative temporary neurological deficits in adults with moyamoya disease. Surg Neurol 69:281-287, 2008

17. Sato K, Shirane R, Yoshimoto T: Perioperative factors related to the development of ischemic complications in patients with moyamoya disease. Childs Nerv Syst 13:68-72, 1997

18. Suzuki J, Takaku A: Cerebrovascular "moyamoya" disease. Disease showing abnormal net-like vessels in base of brain. Arch Neurol 20:288-299, 1969

19. Takase K, Kashihara M, Hashimoto T: Transcranial Doppler ultrasonography in patients with moyamoya disease. Clin Neurol Neurosurg 99 (Suppl 2):S101-S105, 1997

20. Wang Y, Chen L, Wang Y, Pan H, Wang Y, Xu B, et al: Hemodynamic study with duplex ultrasonography on combined (direct/indirect) revascularization in adult moyamoya disease. J Stroke Cerebrovasc Dis 23:2573-2579, 2014

\section{Disclosures}

The authors report no conflict of interest concerning the materials or methods used in this study or the findings specified in this paper.

\section{Author Contributions}

Conception and design: Kim, Jo, Yeon, Hong. Acquisition of data: Cho, Jo, Yu. Analysis and interpretation of data: Kim, Cho, Jo, Yeon, Hong. Drafting the article: Kim, Cho, Jo. Critically revising the article: Kim, Cho, Jo. Reviewed submitted version of manuscript: Kim, Cho, Jo, Yeon, Hong. Approved the final version of the manuscript on behalf of all authors: Kim. Statistical analysis: Cho, Jo. Administrative/technical/material support: Kim, Yu. Study supervision: Kim.

\section{Correspondence}

Jong Soo Kim, Department of Neurosurgery, Samsung Medical Center, Sungkyunkwan University School of Medicine, 81 Irwon-ro, Gangnam-gu, Seoul 135-710, Korea. email: jsns.kim@ samsung.com. 